\title{
La radio estudiantil como estrategia didáctica innovadora
}

Student radio as an innovative learning strategy

\section{Volumen 17, Número 3 \\ Setiembre-Diciembre}

pp. 1-32

Este número se publica el $1^{\circ}$ de setiembre de 2017

DOI: http://dx.doi.org/10.15517/aie.v17i3.30098

\author{
Carlos Araya-Rivera
}

Revista indizada en REDALYC, SCIELO

Revista distribuida en las bases de datos:

LATINDEX, DOAJ, REDIB, IRESIE, CLASE, DIALNET, SHERPA/ROMEO, QUALIS-CAPES, MIAR

Revista registrada en los directorios:

ULRICH'S $, \underline{R E D I E}, \underline{\text { RINACE}}, \underline{\text { OEI }}, \underline{\text { MAESTROTECA }}, \underline{\text { PREAL, }}$ 


\title{
La radio estudiantil como estrategia didáctica innovadora
}

\author{
Student radio as an innovative learning strategy
}

\section{Carlos Araya-Rivera ${ }^{1}$}

\begin{abstract}
Resumen: El empleo de los medios de comunicación colectiva como espacios de aprendizaje ha sido poco estudiado, especialmente en el contexto costarricense. La radio en particular ha sido más apreciada como objeto de estudio que como estrategia didáctica. En este artículo se reseñan los principales resultados de un proyecto de investigación desarrollado en el periodo 2012-2014 acerca del papel de la radio estudiantil como estrategia didáctica. Se utilizó un enfoque metodológico cuantitativo y un diseño no experimental transeccional de tipo secuencial exploratorio. El trabajo de campo se llevó a cabo en dos fases: un inventario de emisoras estudiantiles del Gran Área Metropolitana de Costa Rica y el diseño y ejecución de un Taller de Producción Radiofónica Estudiantil. Para el inventario se aplicó un cuestionario a una muestra censal (405 colegios), que incluyó ítems de respuesta abierta y cerrada, con el fin de recabar información de los centros educativos y las emisoras estudiantiles. El taller se llevó a cabo en dos colegios académicos diurnos urbanos, con la participación de 22 adolescentes que cursaban del $7^{\circ}$ al $12^{\circ}$ nivel de Enseñanza Secundaria, a quienes se aplicaron rúbricas de autoevaluación y coevaluación. Se encontraron 13 experiencias de radio estudiantil en igual número de colegios aunque al finalizar el estudio la mayoría se había cerrado. Luego de la actividad de aprendizaje y la aplicación de rúbricas, el estudio muestra que el grupo de participantes tuvo muy buen desempeño general de la estrategia didáctica. Los resultados indican que esta estrategia podría favorecer el fortalecimiento de valores, la inclusión social y la prevención de problemáticas sociales en la población adolescente, así como el desarrollo de competencias genéricas que pueden brindar insumos importantes para su futuro laboral y profesional.
\end{abstract}

Palabras clave: radio educativa, estrategia de enseñanza, educación sobre medios de comunicación, educación secundaria

Abstract: Use of mass media as learning spaces has not been studied at all, especially in Costa Rican context. Particularly, radio has been used more as an object of study than a learning strategy. This article analyses the role of student radio as a learning strategy over a three-year period (2012-2014). A quantitative methodological approach was used as well as a non-experimental cross-sectional sequential exploratory design. The field work was carried out in two phases: an inventory of student radio stations in the Greater Metropolitan Area of Costa Rica and a Student Radio Production Workshop. For the inventory, a survey was applied to 405 secondary schools, to gather information from schools and student radio stations. The workshop was performed in two urban academic schools, with the participation of 22 teenagers from 7th to 12th grade, to whom self- and co-assessment ratings instruments were applied. Thirteen student radio experiences were found in the same number of schools, although most of them had closed at the end of the study. After the learning activity and the application of instruments, the findings indicates that participants had a very good overall performance of the learning strategy. Results reveals that this strategy could favor the strengthening of values, social inclusion and prevention of social problems in young people, as well as the development of generic competences that can provide important inputs for their future professional.

Keywords: educational radio, teaching strategies, media education, secondary education

\footnotetext{
${ }^{1}$ Catedrático de la Escuela de Ciencias de la Comunicación Colectiva, profesor colaborador de la Escuela de Bibliotecología y Ciencias de la Información e investigador del Instituto de Investigación en Educación, Universidad de Costa Rica. Magister en Educación con acentuación en Procesos de EnseñanzaAprendizaje, Instituto Tecnológico y de Estudios Superiores de Monterrey, México. Magister en Comunicación, Universidad de Costa Rica. Orcid: http://orcid.org/00000002-4450-1902
}

Dirección electrónica: carlos.araya@ucr.ac.cr

Artículo recibido: 21 de junio, 2016

Envío a corrección: 15 de noviembre, 2016

Aprobado: 31 de julio, 2017 


\section{Introducción}

Desde mediados del siglo XX y en el contexto de la sociedad de la información y el conocimiento, los medios de comunicación han sido empleados como recursos de apoyo en los procesos de enseñanza y aprendizaje. En todos los niveles educativos, desde la educación inicial hasta la superior, existen experiencias de utilización de medios de comunicación que, por lo general, se han enfocado en los propios medios y en sus mensajes como recursos didácticos en sí mismos, elaborados principalmente por docentes, profesionales y organizaciones de comunicación.

Periódicos, revistas, programas de radio y televisión, mensajes multimedia y de Internet, se han utilizado más como objetos de estudio o recursos educativos de apoyo, que como medios de comunicación diseñados y producidos por los propios estudiantes. En el caso de la radio, el medio ha servido como canal transmisor de contenidos educativos, articulados como currículum (por ejemplo, en la educación a distancia) o sin estar integrado a algún plan de formación. De ahí el interés por investigar la radio estudiantil como una estrategia didáctica y su papel como espacio de aprendizaje en sí misma.

La importancia de investigar esta temática se encuentra en la naturaleza del medio radiofónico y sus posibilidades educativas, lo cual ha sido documentado y analizado por distintos autores alrededor del mundo. Gascón (1991, p. 8) afirma que la radio contribuye a que las personas tomen decisiones por sí mismas y que aprendan por su propia cuenta, mientras que Kaplún (1999, p. 24) recuerda que las emisiones radiofónicas educativas son tanto aquellas que alfabetizan y difunden conocimientos elementales, como aquellas que favorecen la comunicación de valores, la promoción y el desarrollo integral de las personas y las comunidades. Rodero (2008, pp. 105-106) habla de una "educación auditiva" que se alcanza gracias a la radio, pues el medio capacita para la expresión oral, beneficia la escucha, estimula la imaginación y permite comprender las cualidades y la tipología del sonido.

El uso de la radio como espacio para enseñar y aprender se evidencia en los casos de las radios estudiantiles de Estados Unidos, Colombia, Venezuela, Argentina, Chile y México. En otros continentes también aparecen experiencias de emisoras que siguen esta orientación, y tanto en Europa como en Estados Unidos existen trabajos académicos sobre radios estudiantiles universitarias, entre los cuales destacan los de Sauls (1995a; 1995b; 1998; 2000; 2001), quien valora de manera positiva el impacto de estos medios en la comunidad estudiantil. En la región latinoamericana, Szyszko, Neri y Cataldi (2010) 
consideran que el uso de la radio por Internet y la elaboración de programas radiofónicos permiten abordar de manera más amplia los saberes incluidos en el currículum oficial, por lo cual las y los estudiantes pueden estudiar los temas desde distintas perspectivas. Por su parte, Cárdenas (2008) asegura que la radio escolar es un híbrido entre la radio comunitaria y la radio educativa, con la cualidad de que es producida por niños, niñas y jóvenes en proceso de formación.

Resulta pertinente la observación que hace Zabalza (2009, p. 81) en cuanto a incluir actividades extraescolares dentro del currículum oficial. Al reflexionar si estas actividades deberían ser controladas por el centro educativo, el autor considera que la iniciativa debe corresponder a los propios estudiantes. Desde luego, estas propuestas pueden ser apoyadas por las instituciones, con lo cual es posible obtener beneficios como aportes en contenido y oportunidades de experiencia, entre otros. Es decir, una radio estudiantil no siempre tiene que estar integrada al currículum, pero esto no significa que tenga que competir con él; la oportunidad se abre, entonces, para que lo complemente y fortalezca, y en opinión de Zabalza, el reto es emplear curricularmente el entorno.

En el contexto costarricense, es relevante estudiar la temática pues existen muy pocos trabajos académicos acerca del empleo de medios de comunicación como espacios de aprendizaje, y en particular la valoración del uso de la radio en los centros educativos. En uno de los primeros estudios realizados sobre radio estudiantil en Costa Rica (Araya y Montero, 1997), se indica que en la década de 1990, las prácticas estudiantiles en medios de comunicación se criticaban y observaban con preocupación debido a la formación universitaria en Comunicación vigente en aquella época, que ponía énfasis en el producto (el mensaje) sobre el proceso (la producción o elaboración del mensaje).

Una de las experiencias documentadas es la de Proyecto CONTRASTES, una comunidad de aprendizaje de la comunicación estudiantil que tiene su sede en la Universidad de Costa Rica y que cuenta con más de 25 años de existencia (Araya-Rivera, 2012). En este proyecto participan estudiantes y profesionales jóvenes de distintas disciplinas universitarias, quienes en forma voluntaria diseñan, producen y publican distintos medios y espacios de comunicación, tales como programas radiofónicos, un sitio web, una radio estudiantil digital y campañas de promoción de estos medios de comunicación. Desde el 2009, Proyecto CONTRASTES también lleva a cabo un programa de formación y promoción en radio estudiantil en centros de educación secundaria pública de Costa Rica, en el que han participado 14 centros educativos en actividades formativas enfocadas en el 
diseño y el desarrollo de emisoras estudiantiles. El objetivo de esta iniciativa es promover la creación de una red nacional de radios estudiantiles en Costa Rica, perfiladas como espacios de aprendizaje y de apoyo al currículum de la enseñanza secundaria pública del país.

Aprovechando la experiencia de la iniciativa mencionada, desde la perspectiva académica, interesa estudiar cómo los jóvenes aprenden a producir mensajes radiofónicos y cómo esta estrategia didáctica podría contribuir con el fortalecimiento de valores, la inclusión social y la prevención de problemáticas sociales, como las adicciones, la violencia juvenil y la deserción escolar. Es importante aclarar que se empleará el concepto de estrategia didáctica definido por Feo (2010, p. 222):

Procedimientos (métodos, técnicas, actividades) por los cuales el docente y los estudiantes, organizan las acciones de manera consciente para construir y lograr metas previstas e imprevistas en el proceso enseñanza y aprendizaje, adaptándose a las necesidades de los participantes de manera significativa.

Según el autor, las estrategias didácticas pueden ser de enseñanza, instruccionales, de aprendizaje o de evaluación, lo que depende del actor educativo que las lleve a cabo.

Así las cosas, el trabajo que se presenta a continuación se basa en los hallazgos más relevantes del proyecto de investigación Análisis y asesoría académica de medios radiofónicos estudiantiles como espacios de aprendizaje, desarrollado entre el 2012 y el 2014 en la Escuela de Ciencias de la Comunicación Colectiva de la Universidad de Costa Rica y que, a su vez, generó la tesis presentada por el autor para la Maestría en Educación del Instituto Tecnológico y de Estudios Superiores de Monterrey, México (2015). A continuación, se presentarán algunos elementos conceptuales acerca de la radio estudiantil, el estado actual de este tipo de emisoras en Costa Rica, su valoración como estrategia didáctica en dos grupos de estudiantes de enseñanza secundaria y se emitirán algunas reflexiones en torno al valor de la herramienta tecnológica de la radio como apoyo a los procesos de aprendizaje.

\section{Breve referente teórico}

Desde los experimentos de Marconi hasta la transmisión digital, en poco más de un siglo, la radio ha logrado distinguirse en las preferencias del público. La radio se adapta con mayor velocidad que otros medios a las desafiantes condiciones del mercado y cada día 
cautiva a su audiencia para que mantenga o aumente su atención, al mismo tiempo que complementa la información de la prensa, la televisión y la Internet (Araya-Rivera, 2005).

En la edición electrónica del Diccionario de la Lengua Española en la web, la Real Academia Española (2014) señala que radiodifusión es la "transmisión pública de programas sonoros a través de las ondas hercianas". Es decir, el medio de comunicación emplea la radiofrecuencia para transportar el sonido en sus diversas manifestaciones (voces, música, efectos sonoros, silencio y ambiente), con lo cual construye sentido para una audiencia.

En el contexto costarricense existe muy poca literatura acerca del concepto de radio estudiantil, a diferencia de otros países latinoamericanos, Estados Unidos y Europa donde ha habido mucha más investigación y experiencia sobre este campo. En Estados Unidos, Samuel J. Sauls (2001, p. 6) considera que la radio estudiantil típica no tiene objetivos comerciales y es administrada por un departamento académico. El autor estima que las funciones de la emisora podrían referirse a proveer un entorno con estándares de la industria, en el cual los y las estudiantes puedan aprender las diversas facetas de la radiodifusión, servir al interés público, brindar información útil a la comunidad y ofrecer una programación alternativa y poco común en el mercado radiofónico.

La radio estudiantil se conoce más como formato no comercial que incluye géneros musicales alternativos y programas elaborados por estudiantes universitarios. En este caso, es denominado college radio en Estados Unidos, student radio en Inglaterra y campus radio en el resto de Europa. La tendencia en Estados Unidos y Europa es que la emisora sea totalmente programada y administrada por los propios estudiantes (Araya-Rivera, 2005; 2009). No obstante, en el ámbito latinoamericano se utiliza con mayor frecuencia el concepto de radio escolar para denominar a aquella emisora producida por estudiantes de enseñanza primaria y secundaria, sin importar el grado de complejidad técnica del medio. Es decir, esta radio puede trabajar con un equipo básico de un micrófono, mezcladora de audio y altoparlantes interconectados en los pasillos del centro educativo, o con un sistema basado en web para transmisión con streaming, entre muchas otras posibilidades.

En cambio, el término "radio universitaria" se emplea para las emisoras de las instituciones de educación superior, aunque la mayoría de estas radios responde al enfoque académico y tradicional de los centros públicos y privados, y por lo tanto, no son necesariamente de carácter estudiantil en el sentido norteamericano o europeo. El término genérico ofrece una mejor opción para aquellas personas que se resisten al uso del 
concepto "estudiantil" por su posible connotación negativa que remite a lo poco serio e informal.

Desde otra perspectiva, Perona (2009, p. 109) agrupa en la categoría de emisoras de centros educativos a todas las radios que han aparecido en estos centros, sean de nivel primario, secundario o universitario. Dice el autor que mientras que las emisoras de escuelas y colegios están adaptadas a cada etapa formativa y con contenidos integrados al currículum de Lengua, Conocimiento del Medio y Música, las radios universitarias favorecen la creación de contenidos con todo tipo de géneros y temas, y constituyen una alternativa a las programaciones de las estaciones comerciales.

La traducción al castellano de la denominación "student radio" (radio estudiantil o radio de estudiantes) se aprecia desde lo vivencial de sus productores. En otras palabras, el significado de la emisora se construye desde las experiencias de vida del ser estudiante (Araya-Rivera, 2005-2006), enfocado hacia la actitud de estudiar y aprender, que no se limita a un periodo determinado de la vida. Debido a que se estima pertinente destacar la característica principal de estos medios de comunicación (el diseño y la producción de programas por parte de estudiantes) como estrategia didáctica innovadora, se utilizará el término radio estudiantil para denominar tanto las emisoras de escuelas y colegios como de universidades que sigan el formato de producción de programas de radio estudiantil. Así las cosas, y con base en la sistematización realizada en trabajos previos (Araya-Rivera, 20052006; 2009; 2012 y 2015), se formula la siguiente definición:

La radio estudiantil es un medio de comunicación radiofónica diseñado, gestionado y producido completamente por estudiantes voluntarios de un centro de enseñanza, con contenidos de interés para esta población, que constituye un espacio de experimentación y aprendizaje de la disciplina de la radio y la comunicación, y por lo tanto, en espacio de construcción del conocimiento. Por lo general, cuenta con una asesoría académica permanente, formal o no formal, desempeñada por una o más personas docentes del centro educativo, y cuya labor principal es la de dar acompañamiento y continuidad a los procesos de formación, gestión y operación diaria del medio.

La literatura consultada muestra similitudes entre la definición anterior y el concepto de radio escolar utilizado en América Latina. Sin embargo, hay que observar que en las radios escolares la mayoría de las veces existe un docente o grupo de docentes que realiza el diseño, la planificación y la gestión. Este rasgo se comprende por cuanto la población que 
participa en estas últimas emisoras (niñas y niños) aún no ha desarrollado un alto dominio de las competencias de autorregulación y trabajo en equipo, que son fundamentales para el desempeño de la estrategia didáctica de radio estudiantil.

Kiewe (1987) destaca que, en Estados Unidos es normal considerar la radio estudiantil como una extensión del plan de estudios de la carrera universitaria de Comunicación. No obstante, el autor también plantea la operación de la emisora "como espacio de práctica de comunicación interpersonal y grupal, por lo cual las actividades cotidianas incluyen temas como toma de decisiones, resolución de conflictos, asertividad, confianza y otras habilidades de comunicación efectiva" (p. 6). Según el autor, la radio estudiantil ofrece una alternativa más involucrada e integrada de aprendizaje experiencial.

En un trabajo encargado por las radioemisoras de la Universidad de Costa Rica, Van der Graaf (2001) analizó en forma exploratoria las características de 11 radios estudiantiles universitarias de España, Bélgica, Gran Bretaña y Francia. La investigadora encontró coincidencias en ellas, en cuanto a que operan como espacios de formación, información, de encuentro y diversión, y que son importantes para que el estudiantado aprenda a trabajar en radio de manera profesional, al mismo tiempo que tienen un medio para expresarse y ser escuchados. Por lo tanto, la libertad de expresión y opinión tiene un lugar relevante en la radio estudiantil, ya que es indispensable para una buena comprensión entre los jóvenes y la comunidad.

Para Perona (2002), la emisora escolar permite:

- "Fomentar y reforzar el trabajo en equipo.

- Potenciar la iniciativa y la capacidad creadora del profesorado involucrado en el proyecto.

- Mejorar la expresión oral y escrita entre los estudiantes, así como la utilización de los signos de puntuación.

- Aumentar de forma significativa el uso de la biblioteca.

- Favorecer la integración del alumno, aproximándolo a su entorno.

- Desarrollar una nueva forma de educar: activa, abierta a la vida, democrática, crítica y solidaria.

- Dinamizar la comunicación en la comunidad escolar". (párr. 4) 
Por su parte, Sauls (2001, pp. 6-8) menciona una consulta realizada vía electrónica en el 2000, por Will Robedee de Rice University en Houston, en cuanto a las posibles estructuras y modelos que puede seguir una emisora estudiantil (vea Tabla 1).

Tabla 1

Clasificación de las radios estudiantiles en Estados Unidos

\begin{tabular}{|c|c|}
\hline Categoría & Descripción \\
\hline $\begin{array}{l}\text { Modelo del asesor } \\
\text { académico }\end{array}$ & $\begin{array}{l}\text { Los y las estudiantes operan la estación y obtienen apoyo y asesoría de } \\
\text { un profesional o un asesor académico. Rara vez o nunca, el } \\
\text { asesor toma decisiones en relación con la política y operación de la } \\
\text { radio. }\end{array}$ \\
\hline Modelo estudiantil & $\begin{array}{l}\text { Los y las estudiantes operan por completo la emisora. Por lo general, se } \\
\text { cuenta con un asesor, que no muestra una supervisión directa real, } \\
\text { aunque tal vez exista por escrito. El gobierno estudiantil podría tener } \\
\text { algún papel en la emisora. Este modelo se ubica por lo general en el } \\
\text { área de asuntos estudiantiles de la Universidad. }\end{array}$ \\
\hline $\begin{array}{l}\text { Modelo del } \\
\text { administrador }\end{array}$ & $\begin{array}{l}\text { Existe un administrador o gerente pagado a tiempo completo como } \\
\text { único funcionario. Esta persona docente o administrativa administra la } \\
\text { estación siguiendo la estructura tradicional de radio comercial. }\end{array}$ \\
\hline $\begin{array}{l}\text { Modelo estudiantil- } \\
\text { profesional (Versión 1) }\end{array}$ & $\begin{array}{l}\text { Un equipo de profesionales está a cargo de la administración de la } \\
\text { radio, aunque deja la operación al aire a los estudiantes. Existe tutoría } \\
\text { permanente por parte de los profesionales hacia los estudiantes. La } \\
\text { administración toma las decisiones, aunque consulta a los estudiantes. }\end{array}$ \\
\hline $\begin{array}{l}\text { Modelo estudiantil- } \\
\text { profesional (Versión 2) }\end{array}$ & $\begin{array}{l}\text { Un equipo de profesionales maneja la estación durante una parte del } \\
\text { día y los estudiantes lo hacen en otros momentos. Sin embargo, los } \\
\text { profesionales están completamente a cargo de la política editorial y la } \\
\text { administración. }\end{array}$ \\
\hline $\begin{array}{l}\text { Modelo } \\
\text { exclusivamente } \\
\text { profesional }\end{array}$ & $\begin{array}{l}\text { Como el nombre lo dice, los profesionales están a cargo, aunque } \\
\text { posiblemente hay algunos estudiantes realizando pasantías. }\end{array}$ \\
\hline $\begin{array}{l}\text { Modelo de relaciones } \\
\text { públicas }\end{array}$ & $\begin{array}{l}\text { Es similar al anterior, pero en este caso la emisora es administrada por } \\
\text { la división o departamento de Relaciones Públicas de la universidad. }\end{array}$ \\
\hline $\begin{array}{l}\text { Modelo de radio } \\
\text { pública }\end{array}$ & $\begin{array}{l}\text { Es una emisora afiliada al National Public Radio de Estados Unidos, con } \\
\text { alguna programación local que puede ser producida por profesionales o } \\
\text { por estudiantes. }\end{array}$ \\
\hline $\begin{array}{l}\text { Modelo de laboratorio } \\
\text { (curricular - Versión1) }\end{array}$ & $\begin{array}{l}\text { En muchos aspectos, la radio es una extensión de la clase. Las } \\
\text { políticas, la operación y la participación de los estudiantes están } \\
\text { determinadas por el cuerpo docente de una dependencia académica. }\end{array}$ \\
\hline $\begin{array}{l}\text { Modelo de laboratorio } \\
\text { (curricular - Versión2) }\end{array}$ & $\begin{array}{l}\text { También funciona como una extensión de la clase. Los estudiantes } \\
\text { determinan la programación de la emisora siguiendo directrices } \\
\text { establecidas por la universidad o una de sus dependencias académicas. }\end{array}$ \\
\hline Modelo incorporado & $\begin{array}{l}\text { La emisora es incorporada (es decir, una directiva compuesta por } \\
\text { autoridades de la universidad, profesionales y estudiantes definen las } \\
\text { políticas de gobierno de la emisora). Los y las estudiantes operan la } \\
\text { estación y ejecutan la programación y la gestión diarias. }\end{array}$ \\
\hline
\end{tabular}

Fuente: Sauls (2001, pp. 6-8; adaptado y traducido por Araya-Rivera, 2015, p. 41). 
En cuanto a las opciones de transmisión o publicación, luego de una exploración realizada en la web en la que se revisaron las experiencias de unas 40 radios estudiantiles de colegios y escuelas de España, Argentina, Colombia, México y Chile, se determinaron al menos cuatro modalidades de operación:

- Circuito cerrado de emisión interna: Opera gracias a un equipo de amplificación de audio interconectado con parlantes colocados en los pasillos del centro educativo. La mayoría de las veces, las emisiones se llevan a cabo en los recreos o recesos entre lecciones.

- Radio abierta o de antena: Es una estación radiofónica de baja potencia que funciona al amparo de las legislaciones nacionales que así lo permiten, como ocurrió en Argentina y su Ley de Servicios de Comunicación Audiovisual (2009). Puede transmitir desde algunas horas al día hasta las 24 horas, lo que dependerá de los recursos existentes.

- Streaming por web o Radio Web: Consiste en distribuir audio por Internet de manera continua, para que el contenido pueda ser escuchado al mismo tiempo que se transmite sin necesidad de descargarlo en la computadora del usuario (Middleton, 2006, p.101). En inglés, el término streaming significa "corriente" o "flujo", pues se realiza sin interrupción. En esta modalidad, la radio estudiantil puede operar las 24 horas del día, lo que dependerá de los recursos existentes.

- Podcast: Consiste en un archivo digital de sonido, codificado por lo general en formato MP3 o WMA (Windows Media Audio), y que se puede descargar y escuchar cuando se desea en cualquier dispositivo informático, como una computadora o un teléfono inteligente. El concepto proviene de la combinación del nombre del aparato iPod de la compañía Apple y la terminación cast que en inglés significa difusión o emisión (como en broadcast $=$ radiodifusión). Dice Middleton (2006, p. 110) que en su estructura y tratamiento, los podcast pueden ser similares a programas de radio abierta o bien, seguir un estilo personal, como si fueran blogs hablados. Esta modalidad no es una estación de radio estudiantil en estricto sentido, aunque sí implica una actividad de producción radiofónica desarrollada por los estudiantes, quienes graban sus programas y luego los suben a un sitio web para que los oyentes los descarguen y escuchen.

Como medios de comunicación locales y no masivos, alternativos, no comerciales y que son administrados por centros educativos, las radios estudiantiles pueden ser consideradas como radios comunitarias, ciudadanas o de servicio público. En la literatura 
consultada (en particular Correa Agudelo y López Vargas, 2011; Kaplún, 2001; Montoya y Villa, 2006) se ha encontrado esta relación con toda claridad, pues se considera que la radio estudiantil comparte principios comunitarios como la transmisión de valores, el desarrollo integral de las personas y la comunidad, y la apropiación del lenguaje mediático por parte de grupos sociales vulnerables, de forma que no solo sean receptores o consumidores de mensajes, sino también protagonistas y productores.

Esta perspectiva de la radio estudiantil como derivación de la radio comunitaria se comprende en el marco del derecho a la comunicación, y conlleva una toma de conciencia hacia la modificación de las legislaciones nacionales sobre telecomunicaciones, orientada a establecer reservas de frecuencia en el espectro radioeléctrico que faciliten el acceso a organizaciones educativas y culturales. En este sentido, legislaciones como las de Argentina, Colombia o Ecuador estimulan la discusión acerca de desarrollar experiencias de radio estudiantil en Costa Rica.

Al ser esta temática tan novedosa en el contexto costarricense, en el periodo estudiado no se encontró información en el Ministerio de Educación Pública acerca de las emisoras de centros educativos, sus características, historia, modalidad de funcionamiento, organización y financiamiento ni tampoco su aprovechamiento como estrategia didáctica. Esta fue una de las limitaciones que tuvo la investigación reseñada en este artículo, por lo cual la mayoría de las referencias de la temática proviene de casos extranjeros.

\section{Metodología}

Para llevar a cabo la investigación se optó por un enfoque metodológico cuantitativo y se utilizó un diseño no experimental transeccional, de tipo secuencial exploratorio. El diseño no experimental se seleccionó debido a su flexibilidad, con el fin de comparar los datos obtenidos de la observación del fenómeno y de un registro sistematizado. Además, se utilizó un diseño transeccional para estudiar los datos en un momento determinado. El estudio fue secuencial, de manera que se trabajó en tres etapas:

1. Planteamiento del problema de investigación, revisión de literatura y definición de la metodología de investigación.

2. Trabajo de campo, el cual consistió en recolección de datos de los colegios del Gran Área Metropolitana, diseño, aplicación y evaluación de la estrategia didáctica de radio estudiantil.

3. Análisis e interpretación de los resultados y elaboración del informe de la investigación. 
Por último, y según lo indicado por Hernández, Fernández y Baptista (2010), el alcance de la investigación fue exploratorio, porque el propósito fue examinar un tema que ha sido poco estudiado. Este es el caso de la radio estudiantil en el contexto costarricense.

La etapa de trabajo de campo se llevó a cabo en dos fases: En la primera, y ante la ausencia en Costa Rica de un registro sistematizado de la historia y las características de la radio estudiantil en los colegios de secundaria, se realizó un inventario de las emisoras estudiantiles del Gran Área Metropolitana del país en el periodo 2012-2014. Esta información permitió desarrollar la segunda fase, la cual consistió en el diseño de un plan de formación en producción radiofónica dirigido a estudiantes de enseñanza secundaria, y posteriormente fue evaluada su pertinencia. Dada la naturaleza del estudio de la primera fase, fue necesario optar por un estudio con muestra censal (un total de 405 colegios). Fue así como se decidió realizar esta recopilación de datos en dos periodos: en el primero (2012), se registró la información de los cantones metropolitanos de la provincia de San José, y en el segundo periodo (2013) se recopilaron los datos de las provincias de Heredia, Alajuela y Cartago.

Para registrar la información, se elaboró un instrumento que incluyó ítems de respuesta abierta y cerrada, como ubicación geográfica (provincia, cantón y distrito), naturaleza de la institución (pública, privada o subvencionada), modalidad de enseñanza (técnico/vocacional, artístico, deportivo, académico, experimental, científico, telesecundaria, rural), características de la emisora estudiantil (año de creación, objetivo, modalidad de transmisión, cobertura, programación, temática) y características de las personas participantes (cantidad, nivel educativo, existencia de asesoría académica o persona docente que acompañe el proceso). Este instrumento fue aplicado inicialmente por vía telefónica y cuando no fue posible localizar a la persona encargada, se solicitó, en el centro educativo, un correo electrónico de contacto para enviar el instrumento por esta segunda vía. Luego se procesaron los datos por medio de una hoja de cálculo, para identificar los colegios que tuvieron alguna actividad de producción radiofónica durante el periodo en estudio.

Con base en la información obtenida, para aplicar la estrategia didáctica, se tomó la decisión de seleccionar un colegio que tuviera radio estudiantil y otro que no la tuviera. Por razones de conveniencia, para el primer caso se seleccionó el Liceo San Nicolás de Tolentino, ubicado en San Nicolás (Taras) de Cartago (denominado "Colegio 1") y para el segundo caso se escogió el Liceo de Puriscal, en San José (denominado "Colegio 2"). Ambas son instituciones académicas diurnas públicas que se localizan cerca de las capitales de provincia. La primera es un liceo experimental bilingüe, que ofrece lecciones de idioma 
francés, además del currículum formal, y recibe población en estado de vulnerabilidad, ya que se ubica cerca de una comunidad urbana de clase media-baja y baja. El Colegio 2 cuenta con la opción del programa de Bachillerato Internacional, con lo que el currículum se ofrece durante 6 años o niveles educativos, un nivel adicional al plan regular.

En la segunda fase del trabajo de campo, se optó por una muestra no probabilística definida por conveniencia que, para efectos de la investigación, se determinó con base en los factores señalados por Hernández et al. (2010, p. 394): "capacidad operativa de recolección y análisis, entendimiento del fenómeno y naturaleza del fenómeno bajo análisis". Por tal razón, en esta fase se contó con una muestra de 22 personas, pues dependió de la asistencia de las y los estudiantes que participaron en las fechas en que se aplicaron los instrumentos de medición durante el desarrollo de la estrategia didáctica. Estas personas tenían edades entre los 12 y los 18 años, eran hombres y mujeres de clase socioeconómica baja y media-baja y cursaban del $7^{\circ}$ al $12^{\circ}$ nivel de enseñanza secundaria. Además, residen en las comunidades cercanas a dichos colegios y participaron de forma voluntaria en el estudio.

La estrategia didáctica se aplicó por medio de un Taller de Producción Radiofónica Estudiantil que el investigador impartió con apoyo de personas voluntarias de Proyecto CONTRASTES de la Universidad de Costa Rica. Los contenidos del taller se muestran en la Tabla 2. Esta actividad de aprendizaje tuvo una duración de 28 horas y se desarrolló en 7 sesiones de 4 horas cada una, las cuales se realizaron en los dos centros educativos participantes en el estudio durante el segundo cuatrimestre de 2014.

Tabla 2

Contenidos del Taller de Producción Radiofónica Estudiantil realizado en dos colegios públicos de Costa Rica (Mayo - Agosto de 2014)

1. $\quad$ EI Proyecto CONTRASTES y 11-U. Hacia una radio estudiantil en el colegio.

2. El lenguaje de la Radio (voz, efectos de sonido, música, silencio, ambiente).

3. Redacción y guion para radio.

4. Programación radiofónica - géneros, programas y formatos.

5. Elementos de locución.

6. Entrevista para radio.

7. Planificación de la producción radiofónica.

8. El proceso de la producción radiofónica.

9. La evaluación de la producción radiofónica.

10. Opciones para la puesta en marcha de la emisora: circuito cerrado, streaming, podcast, antena.

Fuente: Araya-Rivera (2015, p. 79). 
El proyecto final de este taller consistió en el diseño y transmisión de prueba de la radio estudiantil del centro educativo que, en el caso de ambos colegios, se realizó por medio de streaming en Internet. En el diseño y la organización del nuevo medio se aplicó el modelo de asesor académico indicado por Sauls (2001, p. 6) expuesto en la Tabla 1, pero con la variación de que el asesor académico tenía mayor influencia y participación en la toma de decisiones. Esta modificación se llevó a cabo debido a las edades de los estudiantes y su falta de dominio de la competencia de autorregulación del aprendizaje, lo cual requirió de mayor liderazgo por parte del asesor académico.

Los contenidos se estudiaron por medio de diversas actividades de aprendizaje, como prácticas de aplicación de los contenidos declarativos y procedimentales, ejercicios en equipo, discusiones grupales, exposiciones magistrales y demostraciones. En cada sesión se efectuó una evaluación formativa entre todos los participantes para corroborar el aprovechamiento del taller.

Por último, se diseñaron rúbricas de autoevaluación y coevaluación que permitieron evaluar la pertinencia y la eficacia de la estrategia didáctica de radio estudiantil. Las rúbricas son instrumentos valiosos para efectos de la investigación debido a su flexibilidad y gran potencialidad didáctica, lo cual es reafirmado por Blanco (2007), Casanellas y Solé (2012), y Pease (2011), entre otros autores. Los instrumentos fueron diseñados para ser autoadministrados y utilizaron una escala de Likert de cinco grados, con una valoración numérica para poder cuantificar los resultados, donde 5 significa el grado más alto de presencia del aspecto y 1 el grado más bajo. A cada punto de la escala numérica corresponde un punto de una escala cualitativa que va de Excelente a Insuficiente, lo que permite valorar el grado de desempeño en la estrategia didáctica.

Los instrumentos se aplicaron en la última sesión del curso. Todos los participantes completaron las rúbricas de autoevaluación, mientras que las de coevaluación solo fueron completadas por los coordinadores de equipo, con el fin de no generar más valoraciones de las necesarias y así evitar la dispersión del estudio. De esta forma, a partir de la propuesta del Análisis de Ritmos de Araya-Rivera (2000), se incluyeron cinco aspectos en las rúbricas:

- Articulación de contenidos declarativos y procedimentales.

- Diseño y ejecución de la producción radiofónica.

- Investigación precisa.

- Cuidado en la calidad del sonido.

- Uso creativo de los recursos del lenguaje radiofónico. 


\section{Resultados y análisis}

\subsection{Censo de radio estudiantil en la GAM de Costa Rica (2012-2014)}

De un total de 405 centros educativos de secundaria del Gran Área Metropolitana, se obtuvo información de 344 colegios en los que se logró contactar a las personas encargadas de las emisoras o a quienes suministraron información acerca del colegio. Es decir, se logró cubrir al $84.9 \%$ de la muestra censal. En el periodo analizado, del total de colegios consultados, se identificaron 13 de ellos con radios estudiantiles o alguna actividad de producción radiofónica estudiantil, como se muestra en la Tabla 3.

Tabla 3

Ubicación de las radios estudiantiles de colegios de la GAM de Costa Rica (2012-2014)

\section{Relativo}

\begin{tabular}{lcc} 
Provincia & Absoluto & \\
\hline San José & 6 & $46.2 \%$ \\
Alajuela & 2 & $15.4 \%$ \\
Cartago & 3 & $23.1 \%$ \\
Heredia & 2 & $15.4 \%$ \\
\hline TOTAL & 13 & $100 \%$
\end{tabular}

Fuente: Elaboración propia

Casi la mitad de las radios estudiantiles se ubican en la provincia de San José, y las demás se distribuyen entre las otras provincias. Mientras que la mayor parte de las emisoras de San José se encuentran en centros educativos de la periferia del casco central (Moravia, Guadalupe, Curridabat, Santa Ana), la mayoría de las estaciones estudiantiles de los otros colegios se ubican en los cantones centrales de las provincias de Alajuela, Cartago y Heredia, como puede apreciarse en la Tabla 4.

El estudio censal permitió descubrir que la mitad de las emisoras están basadas en equipo de amplificación de audio y parlantes instalados en los pasillos de los centros educativos, aunque también crece la tendencia a utilizar la Internet, mediante el uso de streaming o podcast, como se encontró en casi la mitad de los casos. Hay que recordar que, a la fecha, en Costa Rica no es factible la modalidad de radio abierta o de antena, pues la legislación actual no lo permite. Dado que esta investigación se concentró en las radios de los colegios, no se tienen datos en cuanto a emisoras de escuelas primarias. 
De acuerdo con los resultados, la radio estudiantil de colegio más antigua del país es la del Liceo de Paraíso, llamada Radio Base Juvenil y que existe desde 1999. Le sigue la emisora del Instituto de Alajuela, denominada Frecuencia Latina o Frecuencia IDEA y en operación desde el 2002. Llama la atención que, precisamente en el periodo de estudio (2012-2014), surgieron nueve emisoras estudiantiles. Sin embargo, al cierre del análisis, la mayoría de las emisoras o actividades radiofónicas estudiadas fueron canceladas por razones que no pudieron determinarse.

Tabla 4

Características de las radios estudiantiles de colegios en la GAM de Costa Rica (2012-2014)

\begin{tabular}{|c|c|c|c|c|c|c|}
\hline Actividad & Centro educativo & $\begin{array}{c}\text { Naturaleza del } \\
\text { centro }\end{array}$ & $\begin{array}{c}\text { Modalidad de } \\
\text { enseñanza }\end{array}$ & Provincia & $\begin{array}{l}\text { Año de } \\
\text { creación }\end{array}$ & $\begin{array}{c}\text { Modalidad de } \\
\text { transmisión }\end{array}$ \\
\hline $\begin{array}{l}\text { Radio Base } \\
\text { Juvenil }\end{array}$ & Liceo de Paraíso & Público & Académico & Cartago & 1999 & Parlantes \\
\hline $\begin{array}{l}\text { Frecuencia } \\
\text { Latina / IDEA }\end{array}$ & Instituto de Alajuela & Público & Académico & Alajuela & 2002 & Parlantes \\
\hline $\begin{array}{l}\text { Radio Web } \\
\text { Tolentino }\end{array}$ & $\begin{array}{l}\text { Liceo San Nicolás } \\
\text { de Tolentino }\end{array}$ & Público & Académico & Cartago & 2011 & Streaming \\
\hline Rabbit Radio & $\begin{array}{l}\text { Internacional SOS/ } \\
\text { Colegio del Mundo } \\
\text { Unido }\end{array}$ & Privado & Académico & $\begin{array}{l}\text { San } \\
\text { José }\end{array}$ & 2011 & Streaming \\
\hline $\begin{array}{l}\text { Radio CTP } \\
\text { Granadilla }\end{array}$ & $\begin{array}{lr}\text { Colegio } & \text { Técnico } \\
\text { Profesional } & \text { de } \\
\text { Granadilla } & \end{array}$ & Público & Técnico & $\begin{array}{l}\text { San } \\
\text { José }\end{array}$ & 2012 & NS/NR \\
\hline Exprésate & $\begin{array}{l}\text { Colegio Isaac } \\
\text { Martin }\end{array}$ & Privado & Académico & $\begin{array}{l}\text { San } \\
\text { José }\end{array}$ & 2012 & Parlantes \\
\hline $\begin{array}{l}\text { Radio Julio } \\
\text { Fonseca }\end{array}$ & $\begin{array}{l}\text { Liceo Julio Fonseca } \\
\text { Gutiérrez }\end{array}$ & Público & Académico & $\begin{array}{l}\text { San } \\
\text { José }\end{array}$ & 2012 & Parlantes \\
\hline Radio Napo & $\begin{array}{l}\text { Liceo Napoleón } \\
\text { Quesada Salazar }\end{array}$ & Público & Académico & $\begin{array}{l}\text { San } \\
\text { José }\end{array}$ & 2012 & Parlantes \\
\hline Uno Q'Otro & $\begin{array}{l}\text { Colegio } \\
\text { Humanístico } \\
\text { Costarricense } \\
\end{array}$ & Público & Académico & Heredia & 2012 & Podcast \\
\hline Radio Capazu & $\begin{array}{l}\text { Liceo Ing. Carlos } \\
\text { Pascua Zúñiga }\end{array}$ & Público & Académico & Heredia & 2012 & Parlantes \\
\hline Radio Online & $\begin{array}{l}\text { Colegio de Santa } \\
\text { Ana }\end{array}$ & Público & Académico & $\begin{array}{l}\text { San } \\
\text { José }\end{array}$ & 2013 & Streaming \\
\hline Taller de Radio & $\begin{array}{l}\text { Colegio } \quad \text { Santa } \\
\text { Teresa }\end{array}$ & Privado & Académico & Alajuela & 2013 & Streaming \\
\hline Radio COVAO & $\begin{array}{l}\text { Colegio Vocacional } \\
\text { de Artes y Oficios } \\
\text { (diurno) }\end{array}$ & Subvencionado & Técnico & Cartago & 2014 & $\begin{array}{l}\text { Streaming y } \\
\text { parlantes }\end{array}$ \\
\hline
\end{tabular}

Fuente: Elaboración propia 
Se indagó también acerca de las razones por las cuales los centros educativos no tienen emisoras estudiantiles, que en el presente estudio resultó ser significativamente mayor (un $96.2 \%$ en relación con el $3.8 \%$ de los colegios que sí tienen radios). Como puede observarse en el Gráfico 1, la razón principal es la falta de interés de la comunidad educativa del colegio (administrativos, docentes y estudiantes), con un $20 \%$ del total, mientras que también se plantea la falta de recursos (económicos, de infraestructura, de personal capacitado y de tiempo). En el rubro de Otras razones, se menciona la falta de articulación con el currículum, lo cual ya era evidente en los 13 casos de radios estudiantiles mencionados anteriormente.

Gráfico 1

Razones por las cuales los colegios de la GAM no tienen radio estudiantil (2012-2014)

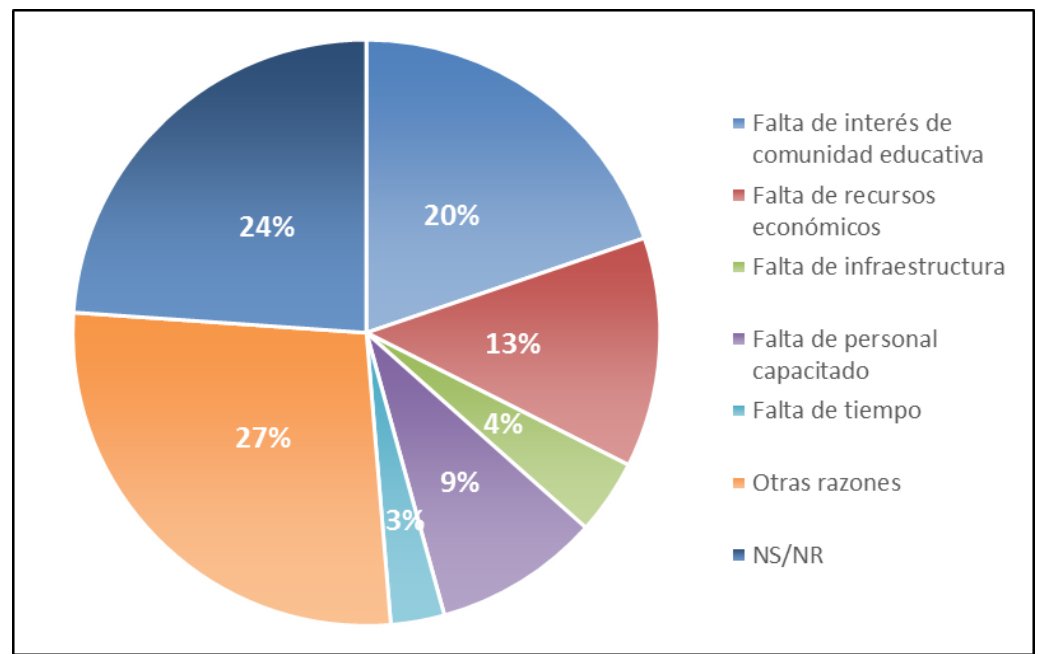

Fuente: Elaboración propia

\subsection{Valoración de la estrategia didáctica de radio estudiantil}

En los dos centros educativos seleccionados, más de las dos terceras partes de las personas consultadas fueron mujeres: el $70 \%$ en el Colegio 1 y $66,7 \%$ en el Colegio 2. Mientras tanto, un poco menos de la tercera parte fueron hombres: $30 \%$ en el Colegio 1 y 33,3\% en el Colegio 2. Al hacer el reclutamiento para el Taller de Producción Radiofónica Estudiantil, quedó en evidencia un mayor entusiasmo en la participación por parte de las mujeres que de los hombres, lo cual podría deberse a la etapa de edad que están experimentando, que se caracteriza por la búsqueda de autoconocimiento, seguridad en sí mismos y manejo de relaciones interpersonales. 
En cada colegio, la población muestra una tendencia hacia los extremos del rango de edades en estudio (13 a 18 años). En el Colegio 1, más de la mitad de los participantes (6 personas) se encuentra en los 13 años, mientras que los demás tienen entre 14 y 17 años, cada uno (4 personas). Mientras tanto, en el Colegio 2 se presenta el otro extremo: la mayoría (5 estudiantes) tiene 16 años y los demás se distribuyen entre los 15, 17 y 18 años de edad. En cuanto al nivel educativo, la mitad del estudiantado está cursando entre $7^{\circ}$ y $9^{\circ}$ año, que corresponde al tercer ciclo de la Educación General Básica en Costa Rica, mientras que la otra mitad cursa entre $10^{\circ}$ y $12^{\circ}$ años, es decir, el ciclo de Educación Diversificada. Más de la cuarta parte de la comunidad estudiantil consultada (27\%) está cursando el $10^{\circ}$ año, mientras que una proporción parecida (23\%) se encuentra en $7^{\circ}$ año. Los grupos etarios no fueron homogéneos, pues dependió del proceso de reclutamiento y selección que se efectuó en cada colegio para el Taller de Producción Radiofónica Estudiantil.

De acuerdo con el planteamiento de la actividad de aprendizaje, en cada institución se formaron tres equipos de trabajo, los cuales responden a la realidad de la industria radiofónica (explicada por Araya-Rivera, 2005), así como a la estrategia didáctica innovadora. Así las cosas, los equipos fueron:

- Producción, que se encarga de la elaboración de los programas radiofónicos.

- Programación, que se encarga de organizar los contenidos en una franja horaria de transmisión.

- Promoción, que se encarga de la divulgación de la emisora y la creación de su identificación o imagen radiofónica.

La mitad de la muestra, es decir 11 participantes, pertenece a los equipos de Producción, mientras que la otra mitad se divide entre los equipos de Promoción y Programación. En el diseño de la actividad de aprendizaje se consideró mantener esta proporción en los equipos, debido a que por su naturaleza, la producción de programas radiofónicos requiere de mayor cantidad de participantes que en el caso de las actividades de promoción o programación.

Como ya se mencionó, la estrategia didáctica de radio estudiantil fue valorada en cinco aspectos: articulación de contenidos declarativos y procedimentales; diseño y ejecución de la producción radiofónica; investigación precisa; cuidado de la calidad del sonido y uso creativo de los recursos del lenguaje radiofónico. En primer lugar, la articulación de contenidos se evidencia como "Muy Buena" en 12 individuos, es decir, más de la mitad de la muestra, y en 6 personas como "Excelente". De estos, es en el equipo de Producción donde se concentra 
la mayoría de las respuestas positivas (ver Gráfico 2). Mientras, la coevaluación de los líderes muestra a sus compañeros con un desempeño "Excelente" en 8 casos y "Muy Bueno" en 7 casos, y de nuevo es el equipo de Producción el que tiene mayoría de respuestas positivas. Sin embargo, hay que notar que en el equipo de Promoción aparecen dos personas con valoración "Regular" y en Producción hay otra persona con "Regular" y otra con "Insuficiente", que puede indicar la necesidad de buscar una mejor articulación entre los contenidos impartidos durante la actividad de aprendizaje (ver Gráfico 3).

Gráfico 2

Autoevaluación del desempeño en articulación de contenidos en la estrategia didáctica de radio estudiantil

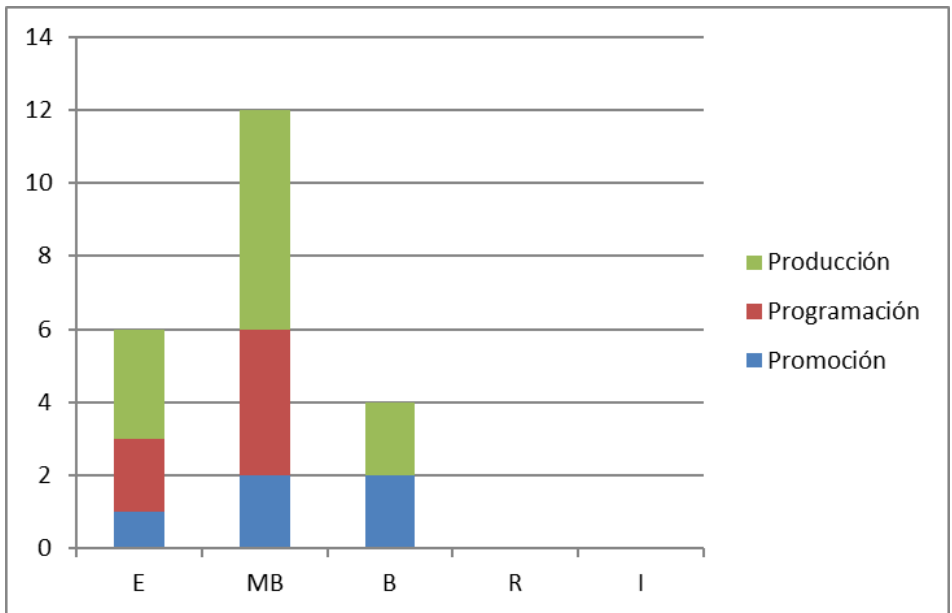

Fuente: Adaptado de Araya-Rivera (2015, p. 102)

Gráfico 3

Coevaluación del desempeño en articulación de contenidos en la estrategia didáctica de radio estudiantil

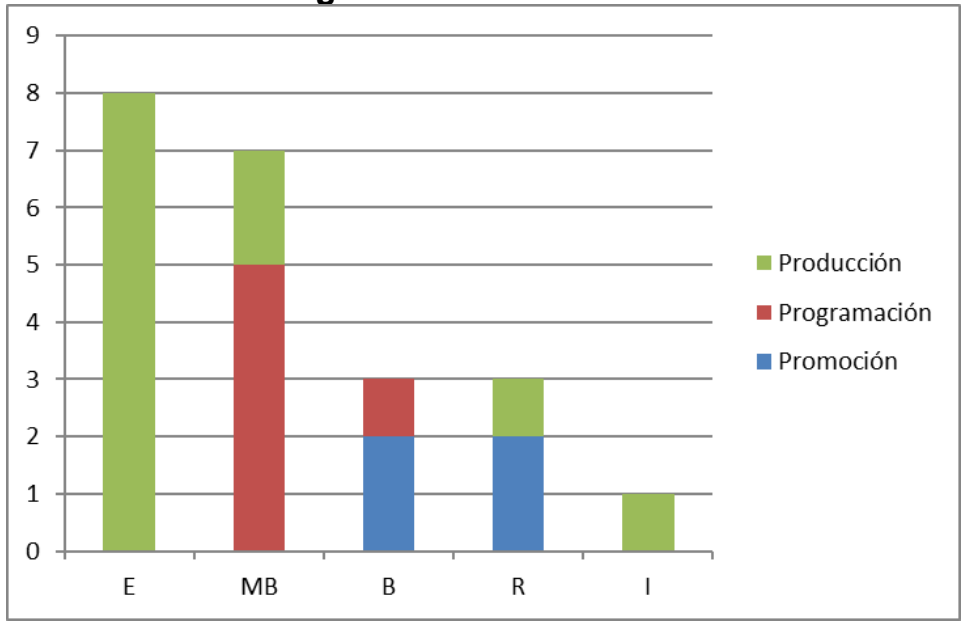

Fuente: Adaptado de Araya-Rivera (2015, p. 102) 
En relación con el desempeño en el diseño y la ejecución de la producción radiofónica, cerca de las dos terceras partes de los consultados (14) expresaron tenerlos en forma "Excelente", a la vez que la tercera parte restante (8) se distribuyó en los otros puntos de la escala (ver Gráfico 4). En la coevaluación, los coordinadores de equipo ubicaron a sus compañeros en el rango de "Excelente" a "Regular", con más valoraciones positivas en el equipo de Producción y con valoraciones intermedias en los otros dos equipos, y hubo tres casos con valoración "Regular": uno en Promoción y dos en Producción (ver Gráfico 5).

Gráfico 4

Autoevaluación del desempeño en producción radiofónica en la estrategia didáctica de radio estudiantil

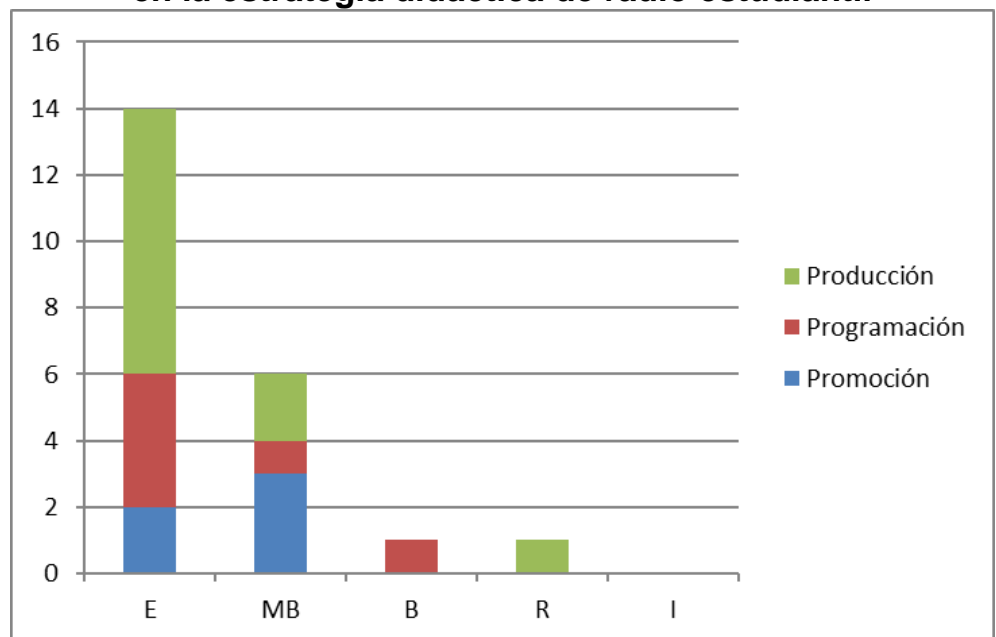

Fuente: Adaptado de Araya-Rivera (2015, p. 103)

Gráfico 5

Coevaluación del desempeño en producción radiofónica en la estrategia didáctica de radio estudiantil

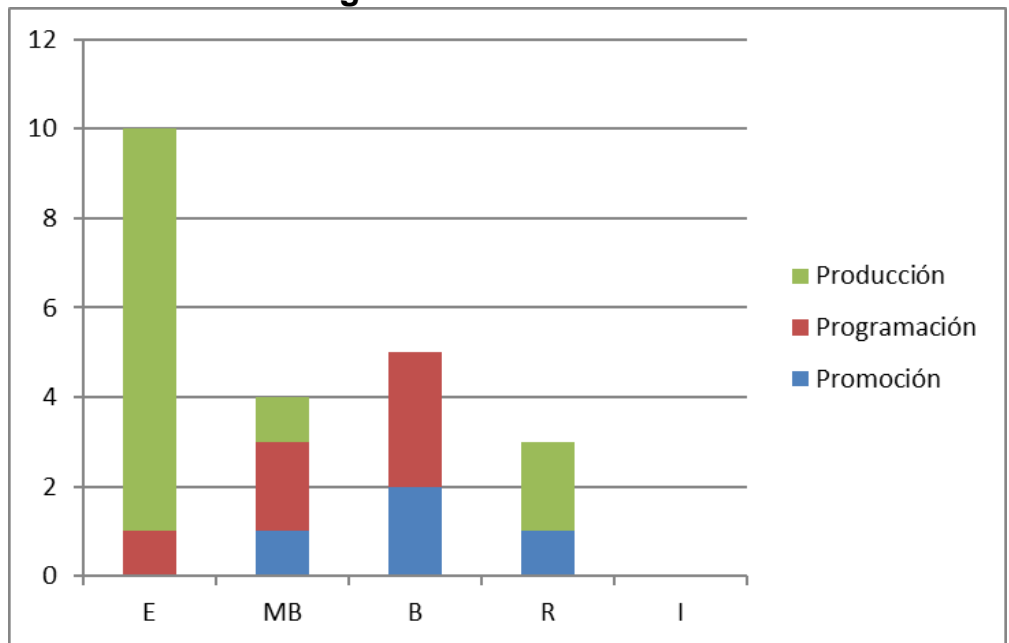

Fuente: Adaptado de Araya-Rivera (2015, p. 103) 
En el aspecto de la investigación precisa, con uso de fuentes confiables, conocimiento del tema y del público, 11 personas valoraron su desempeño como "Excelente", mientras que 9 personas lo consideraron como "Muy Bueno" (ver Gráfico 6). Los líderes de los equipos también siguieron esta tendencia en la coevaluación de pares, pues aparece una mayoría de valoraciones de "Excelente" y "Muy Bueno" (ver Gráfico 7).

\section{Gráfico 6}

Autoevaluación del desempeño en investigación precisa en la estrategia didáctica de radio estudiantil

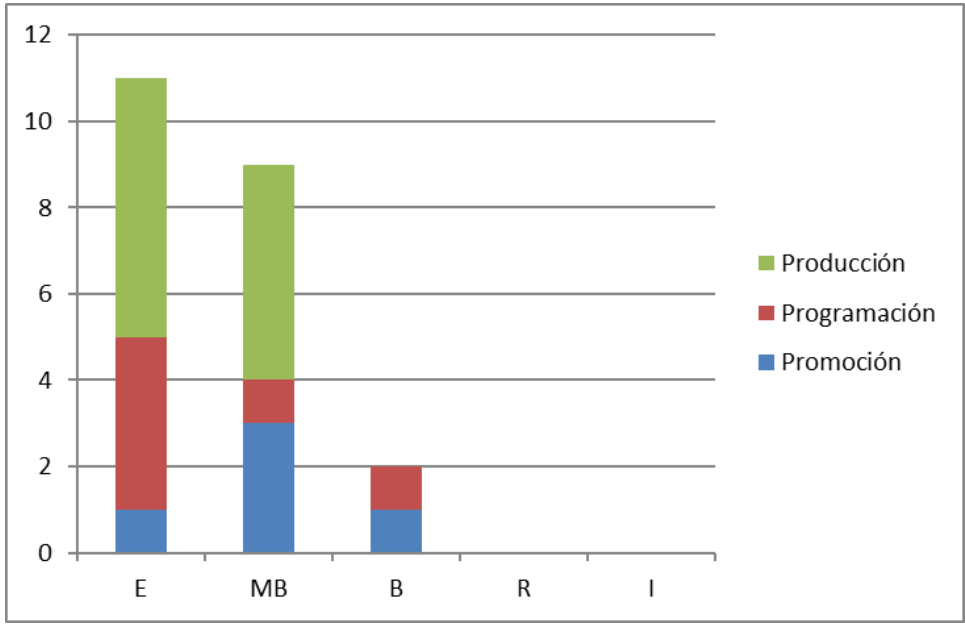

Fuente: Adaptado de Araya-Rivera (2015, p. 104).

Gráfico 7

Coevaluación del desempeño en investigación precisa en la estrategia didáctica de radio estudiantil

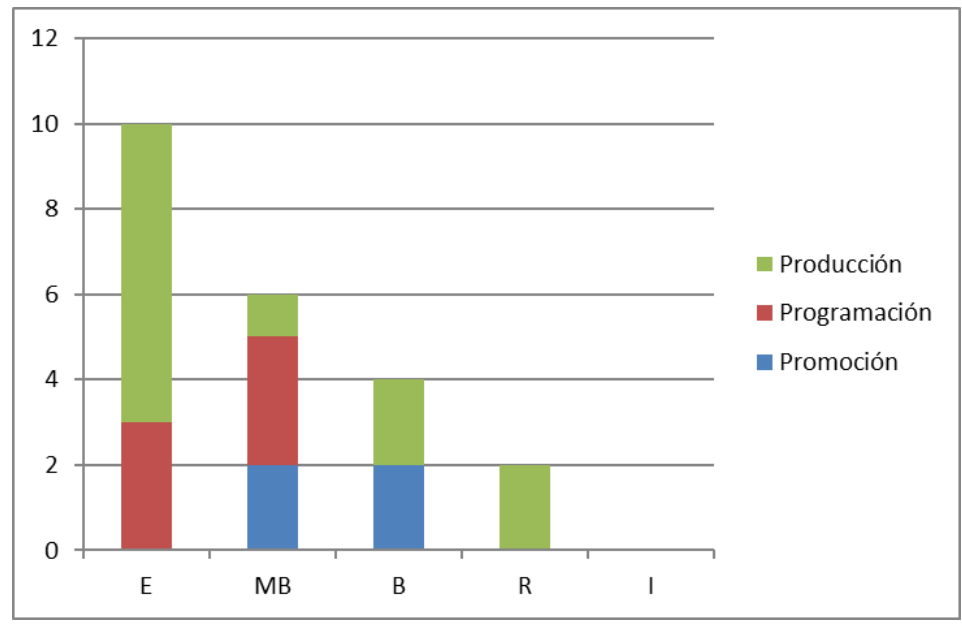

Fuente: Adaptado de Araya-Rivera (2015, p. 104). 
Con el cuidado de la calidad del sonido, cambia la percepción y se encuentra una visión más crítica. En su autoevaluación, la comunidad estudiantil mostró una mayoría de valoraciones de "Muy Bueno" y "Bueno", 10 y 6 personas respectivamente, frente a 5 que expresaron una valoración de "Excelente" y un estudiante que se valoró como "Regular" (ver Gráfico 8). Los líderes coevalúan, en general, de forma positiva a sus compañeros y concentran las valoraciones de "Excelente" y "Muy Bueno" en 16 personas, frente a 6 restantes que se ubican en "Bueno" o "Regular" (ver Gráfico 9).

\section{Gráfico 8}

Autoevaluación del desempeño en cuidado de la calidad del sonido en la estrategia didáctica de radio estudiantil

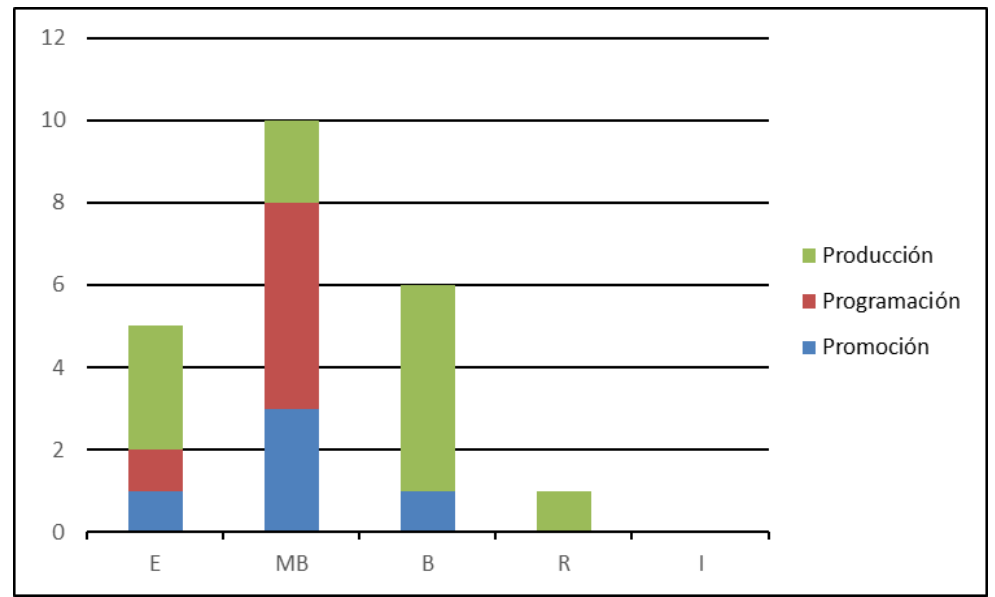

Fuente: Adaptado de Araya-Rivera (2015, p. 105).

Gráfico 9

Coevaluación del desempeño en cuidado de la calidad del sonido en la estrategia didáctica de radio estudiantil

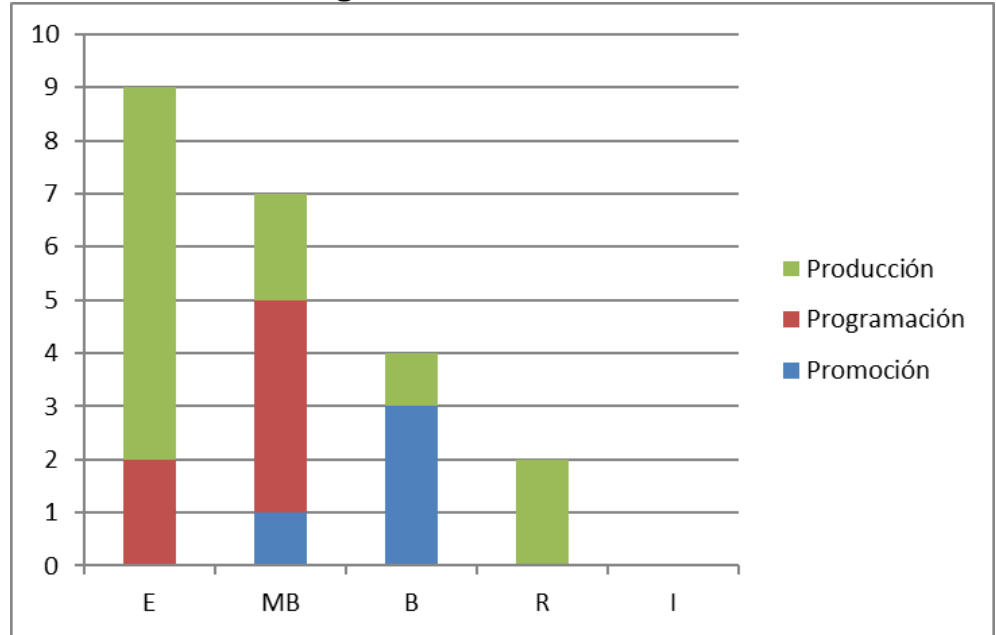

Fuente: Adaptado de Araya-Rivera (2015, p. 105). 
En el último aspecto, uso creativo del lenguaje radiofónico, el estudiantado consideró su desempeño como "Excelente" o "Muy Bueno" en casi todos los casos; es decir, 21 personas (ver Gráfico 10), mientras que en la coevaluación, los líderes estimaron que la mitad de sus compañeros de equipo, 11 estudiantes, tuvieron un desempeño "Bueno" (ver Gráfico 11).

\section{Gráfico 10}

Autoevaluación del desempeño en uso creativo del lenguaje radiofónico en la estrategia didáctica de radio estudiantil

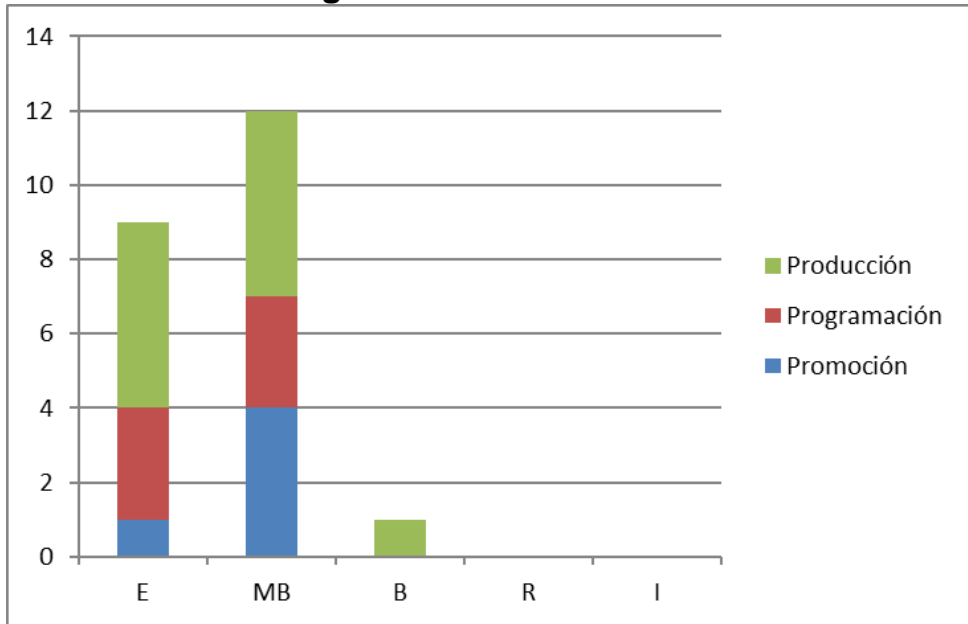

Fuente: Adaptado de Araya-Rivera (2015, p.106).

\section{Gráfico 11}

Coevaluación del desempeño en uso creativo del lenguaje radiofónico en la estrategia didáctica de radio estudiantil

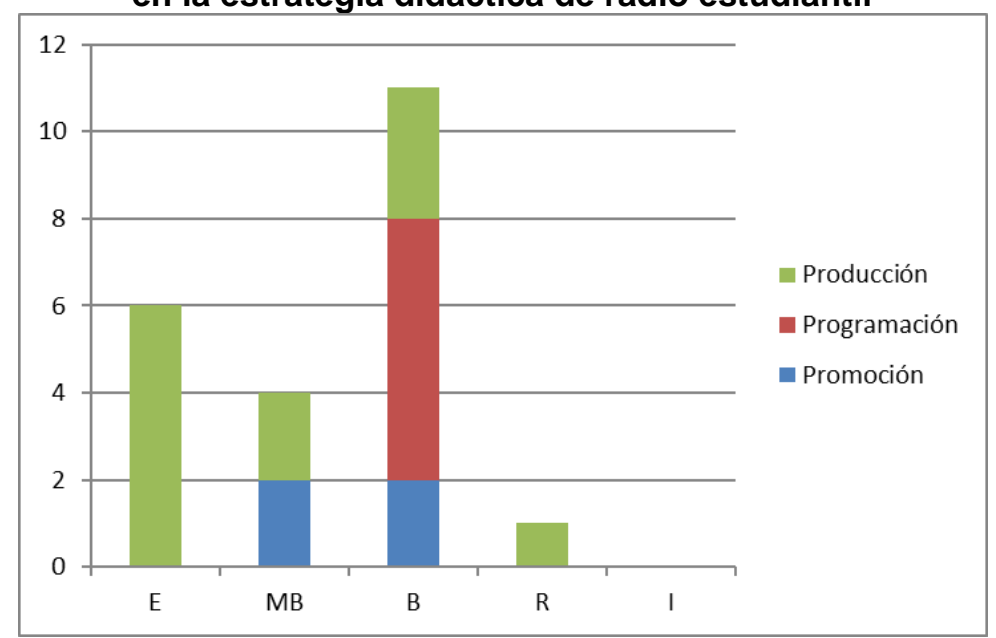

Fuente: Adaptado de Araya-Rivera (2015, p.106). 
Finalmente, los resultados de cada aspecto fueron sumados para establecer un posible grado del desempeño general en la estrategia de radio estudiantil. Así, se obtuvo que 9 personas de Producción recibieron una valoración "Excelente", seguidas por 5 participantes de Programación y 3 de Promoción, para un total de 17 individuos, lo que significa más de la mitad de los consultados (ver Gráfico 12). En la coevaluación de los coordinadores de Programación y Producción, se tiene a 10 personas con un "Excelente", mientras que en Promoción se valoró a los 4 participantes con "Muy Bueno" y "Bueno" (ver Gráfico 13).

\section{Gráfico 12}

Autoevaluación del desempeño general en la estrategia didáctica de radio estudiantil

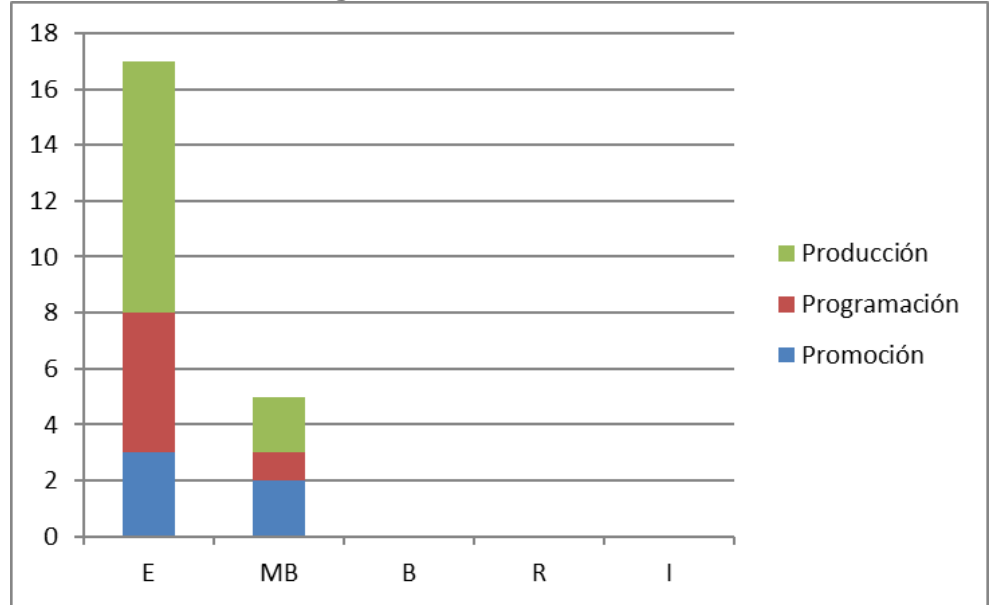

Fuente: Adaptado de Araya-Rivera (2015, p.107).

Gráfico 13

Coevaluación del desempeño general en la estrategia didáctica de radio estudiantil

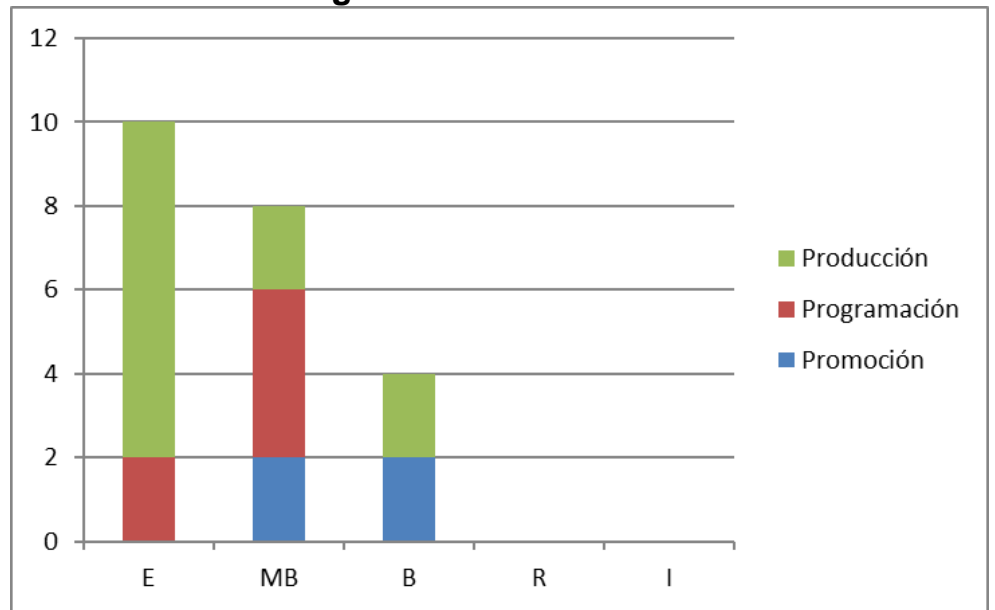

Fuente: Adaptado de Araya-Rivera (2015, p.107). 


\subsection{Análisis de los datos}

El desempeño de los diferentes elementos que conforman la estrategia didáctica en estudio fue sustentado con el planteamiento del Análisis de Ritmos de Araya-Rivera (2000), en relación con el proceso general de la producción radiofónica y en particular con su diseño y ejecución: primero se investiga un tema y se redacta en lenguaje radiofónico, manteniendo cierta cercanía con el público (ritmo interno). Luego, se selecciona la voz o las voces que narrarán el texto, así como los efectos sonoros y la música que completarán el mensaje (ritmo externo), y todo queda enmarcado en la elaboración de un guion, que debe diseñarse con base en un género de la comunicación determinado, como por ejemplo un reportaje, una noticia, una radio-revista o un microprograma.

Inicialmente, se observó una articulación básica de contenidos declarativos y procedimentales que permitió a su vez el diseño y la ejecución de la producción radiofónica propuesta en la actividad de aprendizaje. El desempeño de ambos aspectos fue valorado en los grados "Bueno", "Muy Bueno" y "Excelente", lo que evidenció el interés de los jóvenes en el aprendizaje de una herramienta novedosa y diferente para expresar sus ideas y sentimientos. Así se confirma lo declarado por Montoya y Villa (2006), en cuanto a que la radio estudiantil abre espacios para que los jóvenes conversen acerca de los problemas que los afectan, con lo cual pueden convertirse en perceptores críticos de los medios de comunicación.

Los siguientes aspectos, investigación precisa y cuidado en la calidad del sonido, se observaron en un primer momento con un desempeño "Bueno", pues en el primer caso se requería una mayor criticidad en la selección y uso de las fuentes de información, mientras que en el segundo, faltaba vigilar más la calidad de la grabación. En cuanto a la autovaloración del estudiantado, el desempeño del aspecto de investigación precisa es "Excelente" y el cuidado en la calidad de sonido es "Muy Bueno". Como en los aspectos anteriores, la calificación se sobredimensiona.

El aspecto de la investigación precisa en la producción radiofónica ha sido señalado por diversos autores, entre ellos Alves (1984), para quien la información debe ser completa, imparcial y sin prejuicios. A su vez, Araya-Rivera (2000) había incluido este elemento como parte del Análisis de Ritmos, propuesta conceptual en la que se basa la estrategia didáctica estudiada en este trabajo. La alta valoración del aspecto por parte de los estudiantes más bien llama a reflexionar acerca de la urgencia por promover el uso de estrategias de lectura crítica, con el fin de que valoren la información que reciben o que buscan en fuentes 
populares como Wikipedia o las redes sociales. Esto facilitaría la toma de decisiones y en consecuencia aprenderían a buscar información de mayor calidad, útil, precisa y pertinente.

Con respecto al cuidado en la calidad del sonido, se reconoce un manejo básico de la herramienta de la radio estudiantil, la cual, como ya se mencionó, permite a los jóvenes comunicar sus propuestas y reflexionar sobre sus vidas. Hay que aclarar que en el Taller de Producción Radiofónica Estudiantil se enseñó a las y los jóvenes a trabajar con el equipo tecnológico que tuvieran (por ejemplo, teléfonos celulares, computadores personales y tabletas). Aunque la disciplina de producción radiofónica requiere la mayor calidad posible de grabación de sonido, se entiende que existan condiciones especiales relacionadas con el tipo de equipo al que tienen acceso los estudiantes, y por lo tanto la estrategia didáctica se desarrolló con los recursos disponibles en cada centro educativo. En todo caso, se insistió en la necesidad de obtener la mejor calidad posible de grabación con esos recursos, con el fin de que los jóvenes pudieran utilizar la herramienta de la radio estudiantil y así integrar las Tecnologías de la Información y la Comunicación en su proceso de aprendizaje y adquirir competencias digitales, como lo refieren en sus trabajos Ferrada, Gaete y Sáez (2010) y Szyszko, Neri y Cataldi (2010), entre otros investigadores.

En cuanto al uso creativo de los recursos del lenguaje radiofónico, se muestra un desempeño "Muy Bueno" tanto en la medición realizada con la observación como en la autoevaluación. No obstante, por primera vez en este estudio, aparece una valoración más crítica que considera el desempeño de este aspecto como "Bueno", que si bien no es malo, tampoco es el punto más alto de la escala de medición. Los gráficos 10 y 11 muestran una tendencia distinta a las otras presentadas anteriormente, la cual consiste en que se valora de manera más crítica y menos complaciente que en los otros aspectos.

Con los proyectos finales del taller, el investigador encontró evidencia de uso creativo del lenguaje radiofónico para el nivel y las posibilidades de los equipos de estudiantes, pero también fue notoria la apreciación crítica de los propios jóvenes en cuanto a querer mejorar el trabajo realizado. Dicha apreciación fue manifestada en la última sesión del taller, durante la cual los estudiantes expresaron su interés y compromiso en continuar trabajando el proyecto de radio estudiantil en su colegio.

Para finalizar el análisis del desempeño en la estrategia didáctica, más de la mitad de las personas consultadas obtuvieron "Excelente" en el desempeño general, lo cual fue la tendencia mayoritaria en todos los equipos, con las excepciones ya señaladas. No obstante, el resultado contrasta con la evaluación de pares, pues el equipo de Promoción recibió 
menor calificación que los otros equipos. De igual manera, se observa que el elemento común entre la autoevaluación y la coevaluación del desempeño en la estrategia didáctica de radio estudiantil, es el menor desempeño por parte del equipo de Promoción, comparado con los otros dos equipos. Nuevamente hay que comprender la naturaleza de las tareas de este equipo, las cuales se concentran en el diseño y la producción de la imagen radiofónica de la emisora estudiantil (anuncios promocionales e identificación sonora, por ejemplo), circunstancias propias de la industria que han sido explicadas por Araya-Rivera (2005) y otros autores. En consecuencia, aunque las tareas del equipo de Promoción conllevan más trabajo de planificación que de producción radiofónica concreta, se evidenció una falta de articulación de los contenidos declarativos y procedimentales, por lo que proporcionalmente el equipo de Promoción tuvo un nivel de desempeño menor en la estrategia didáctica que los otros dos equipos.

\section{Conclusiones}

Durante la aplicación de la estrategia didáctica se hizo evidente el interés de las y los jóvenes en el aprendizaje de la radio estudiantil como una herramienta novedosa y diferente para expresar sus ideas y sentimientos, con la cual pueden generar un cambio en su entorno inmediato. El estudiantado valoró la importancia social de este recurso como una oportunidad para aprender y a la vez aportar información útil a sus pares. En ese sentido, resulta valioso comprobar el interés y el compromiso en cuanto a mantenerse como equipo y continuar el proyecto de radio estudiantil en su colegio.

La evaluación del desempeño del estudiantado en la estrategia didáctica incluyó con mayor frecuencia las calificaciones de "Regular" e "Insuficiente" en todos sus componentes, que son el uso creativo del lenguaje radiofónico, el cuidado de la calidad del sonido, la investigación precisa, la producción radiofónica y la articulación de los contenidos declarativos y procedimentales. Los líderes de equipos mostraron mayor criticidad en la evaluación de estos aspectos, y es posible que se deba a que los componentes de la estrategia didáctica de radio estudiantil fueran cercanos a la cotidianidad de los estudiantes.

Siempre en esta línea, cabe mencionar el aspecto de la investigación precisa, que resultó ser muy bien valorado en la autoevaluación y la coevaluación, pero que al triangular los datos se encontró que faltaba mayor criticidad en la selección y uso de las fuentes de información. En ese sentido, la estrategia didáctica de radio estudiantil ofrece un espacio 
adecuado para realizar la reflexión, hacer una lectura crítica de las fuentes y favorecer el aprendizaje de este aspecto.

Al comparar la autoevaluación de los tres equipos de trabajo organizados para el proyecto de la emisora estudiantil en cada colegio, se encontró que, proporcionalmente, el equipo de Programación tuvo mejor desempeño general en la estrategia didáctica, mientras que el equipo de Promoción tuvo menor desempeño general que los otros. Estas diferencias se pueden comprender al tomar en cuenta la naturaleza de las tareas específicas asignadas a cada equipo y los problemas que debieron resolver los estudiantes para poder alcanzar las metas fijadas, según se señaló.

Sin embargo, el hallazgo más relevante de esta comparación se refiere a la forma en que las y los estudiantes de Programación lograron enfrentar los desafíos planteados con el proyecto de radio estudiantil. La coordinación del líder, unida al convencimiento de alcanzar el objetivo colectivo como prioridad antes que las necesidades individuales, así como el compromiso evidenciado por los participantes, contribuyeron a alcanzar las metas propuestas y con ello, un desempeño adecuado en la estrategia didáctica. En el otro extremo, estudiantes de los equipos de Promoción y Producción presentaron un desempeño menor debido a las dificultades asociadas con la falta de liderazgo y de articulación de contenidos declarativos y procedimentales. Por último, se considera que el grupo de estudiantes participantes en esta investigación tuvo un muy buen desempeño general de la estrategia didáctica de radio estudiantil, lo cual beneficia a los jóvenes por cuanto les brinda insumos importantes para su futuro laboral y profesional.

En el contexto costarricense, entre los nuevos aspectos que podrían conocerse mejor, están la localización y las características de las radios estudiantiles que existen en el país que, como se indicó al inicio de este artículo, a la fecha no existe información sistematizada al respecto. Asimismo, sería interesante conocer cómo esas emisoras se integran al currículum escolar de los centros educativos, y si están vinculadas de alguna forma con centros de recursos para el aprendizaje y bibliotecas escolares, que podrían ofrecer espacios adecuados y recursos valiosos para su desarrollo.

También podría proponerse una coordinación entre el Ministerio de Educación Pública de Costa Rica y las universidades estatales, para crear una red de radios estudiantiles de escuelas y colegios del país que sirvan de espacios para el aprendizaje, que se integren al currículum de distintas materias y que podrían promover la formación por competencias. De esta forma, se ofrecería una herramienta útil a niñas, niños y jóvenes que les permitiría 
desarrollar competencias genéricas como el trabajo en equipo, la autorregulación, el pensamiento crítico, la gestión del tiempo, la resolución de problemas, la comunicación verbal oral y escrita y la alfabetización mediática e informacional, entre otras. Con la radio estudiantil, los jóvenes tendrían un valor agregado importante en su formación, pues esta estrategia les permitiría fortalecer valores como la colaboración, la tolerancia y la solidaridad, además de contribuir con la prevención de la deserción escolar, la violencia juvenil, la delincuencia y las adicciones al alcohol y las drogas, entre otras problemáticas sociales.

Una limitación que se tuvo en esta investigación fue el seguimiento de las experiencias de radio estudiantil estudiadas, ya que una vez terminada la actividad de aprendizaje, no fue posible mantener el acompañamiento. Esto supone un riesgo importante para la continuidad de las experiencias, pues cuando concluye el Taller de Producción Radiofónica Estudiantil se depende de los docentes responsables en cada colegio para desarrollar los proyectos de emisoras. Así mismo, en al menos uno de los casos, el proyecto se congeló por cambio de la persona docente que estaba a cargo. Por tal razón, convendría involucrar al menos a dos docentes en cada centro educativo, quienes colaboren como asesores académicos de la radio estudiantil que se pretenda establecer en cada colegio, de manera que se garantice un acompañamiento permanente para las y los jóvenes. En ese mismo sentido, se recomienda la participación de las personas profesionales en Bibliotecología, ya que su formación en Ciencias de la Información y en Educación podría facilitar la consolidación de las experiencias de radio estudiantil desde las bibliotecas de los centros educativos.

Como se trató de una actividad extracurricular, se dependió también del tiempo de docentes responsables y de estudiantes participantes en los colegios, quienes tenían que desarrollar las tareas del curso de radio a la par de sus obligaciones académicas. Por lo tanto, sería conveniente plantear la ejecución de las actividades de formación dentro del horario lectivo, con el fin de garantizar tanto la asistencia como el compromiso de los participantes que algunas veces se vieron afectados en el transcurso de la actividad de aprendizaje.

Enseñar no es una simple transmisión de conocimientos y experiencias, sino que implica facilitar las oportunidades necesarias para que el aprendiz adquiera la habilidad de continuar aprendiendo. Comentando a Heidegger, Bedoya (2008, p. 39) señala que "enseñar no se reduce a lo que acontece en un aula de clase, donde se enfatiza lo que hace -o no hace- un docente que es el que efectivamente enseña". Es decir, el acto educativo no se limita al espacio de aula y existen muchos otros espacios de la vida cotidiana del estudiante 
en que también ocurren actos educativos, lo cual debe llevarnos a reflexionar cuáles podrían ser esos nuevos espacios. En esta investigación se ha demostrado que la radio estudiantil es uno de ellos.

El empleo de estrategias didácticas innovadoras como la radio estudiantil podría facilitar el desarrollo de las competencias genéricas en los y las estudiantes de enseñanza secundaria, siempre y cuando exista el compromiso personal de educadores y educandos en cuanto a aprender y a compartir conocimiento con todos los miembros de la comunidad educativa. Si esto se lograra, la radio estudiantil podría convertirse en un recurso educativo poderoso, como espacio de aprendizaje y de comunicación de ideas, sueños, conocimientos y experiencias que podrían mejorar el mundo en que vivirán las próximas generaciones.

\section{Agradecimientos}

El autor desea reconocer el apoyo de Natalia Aguilar Chinchilla, Diana Carrillo Rosales, Hawi Castañeda Willca, Aarón Chaves González, Francisco Corella Quirós, Jenny García Fernández, Marypaz Morales Cordero, José Enrique Ramírez Chaves, Diego Rivera Vásquez, Luis Sequeira Sandoval y Manrique Vargas Soto, participantes en el Proyecto CONTRASTES y colaboradores del proyecto de investigación 212-B2-013 Análisis y asesoría académica de medios radiofónicos estudiantiles como espacios de aprendizaje, desarrollado en la Universidad de Costa Rica.

\section{Referencias}

Alves, Walter Ouro. (1984). Radio: La mayor pantalla del mundo. Quito, Ecuador: CIESPAL.

Araya-Rivera, Carlos. (2000). El ritmo como instrumento de análisis de programas radiofónicos. Revista Educación, 24(2), 175-182. Recuperado de DOI: http://dx.doi.org/10.15517/revedu.v24i2.482

Araya-Rivera, Carlos. (2005). Manual de producción radiofónica estudiantil (Tesis de Maestría). Universidad de Costa Rica, Posgrado en Comunicación, San José, Costa Rica.

Araya-Rivera, Carlos. (2005-2006). La revolución radiofónica. Revista Girasol, 6-7, 281-288.

Araya-Rivera, Carlos. (2009). Radio estudiantil: programas, audiencias y desafíos. Revista Reflexiones, 88(2), 37-44. $\quad$ Recuperado de http://revistas.ucr.ac.cr/index.php/reflexiones/article/view/11520/10865 
Araya-Rivera, Carlos. (2012). Proyecto CONTRASTES: medios de comunicación estudiantil como espacios de aprendizaje. Revista Educación, 36(1), 83-95. Recuperado de http://dx.doi.org/10.15517/revedu.v36i1.454

Araya-Rivera, Carlos. (2015). Radio estudiantil como estrategia didáctica para desarrollar el trabajo en equipo en estudiantes de secundaria pública de Costa Rica (Tesis de Maestría). Instituto Tecnológico y de Estudios Superiores de Monterrey, Escuela de Graduados en Educación, Monterrey, México.

Araya Rivera, Carlos y Montero Bolaños, Fernando. (1997). La producción de una radiorevista como espacio de práctica para los estudiantes de Comunicación Colectiva de la Universidad de Costa Rica (Tesis de Licenciatura). Universidad de Costa Rica, Escuela de Ciencias de la Comunicación Colectiva, San José, Costa Rica.

Bedoya, José Iván. (2008). Pedagogía ¿enseñar a pensar? Reflexión filosófica sobre el proceso de enseñar. Bogotá, Colombia: Ecoe Ediciones.

Blanco Blanco, Ángeles. (2007). Las rúbricas: un instrumento útil para la evaluación de competencias. En: Leonor Prieto (Coord.), La enseñanza universitaria centrada en el aprendizaje. Estrategias útiles para el profesorado (pp. 171-188). España: Octaedro Editorial.

Cárdenas Mora, Ángela Marcela. (2008). Radio Creativa en la radio escolar. Vía libre a la imaginación y la fantasía (Tesis de grado). Pontificia Universidad Javeriana, Facultad de Comunicación y Lenguaje, Bogotá, Colombia. Recuperado de http://www.javeriana.edu.co/biblos/tesis/comunicacion/tesis83.pdf

Casanellas Chuecos, Montserrat y Solé Català, Marina. (junio, 2012). La competencia transversal de trabajo en equipo. Instrumentos para su implementación y evaluación. Trabajo presentado en la III Jornada sobre Docencia del Derecho y Tecnologías de la Información y la Comunicación. Barcelona, España: Universitat Oberta de Catalunya. Recuperado de http://www.uoc.edu/symposia/dret tic2012/pdf/3.4.casanellas$\underline{\text { montserrat-y-sole-marina.pdf }}$

Correa Agudelo, Luis Felipe y López Vargas, Andrés David. (2011). La radio escolar como una estrategia de enseñanza-aprendizaje en el Colegio Hernando Vélez Marulanda (Tesis de Licenciatura). Universidad Tecnológica de Pereira, Facultad de Educación, Pereira, Colombia.

Ferrada Montecinos, Yasna Patricia, Gaete Saldías, Javiera María Esperanza y Sáez Beltrán, Domingo Wilfredo. (2010). Radio escolar online, exploración en informática educativa. Razón y Palabra, 15(74). Recuperado de http://www.redalyc.org/articulo.oa?id=199516111024

Feo Mora, Ronald J. (2010). Orientaciones básicas para el diseño de estrategias didácticas. Tendencias pedagógicas, (16), 221-236.

Gascón Baquero, María Carmen. (1991). La radio en la educación no formal. Barcelona, España: Ediciones CEAC, S.A. 
Hernández Sampieri, Roberto, Fernández-Collado, Carlos, y Baptista Lucio, Pilar. (2010). Metodología de la investigación. México, D.F.: McGraw-Hill.

Kaplún, Mario. (2001). A la educación por la comunicación. La práctica de la comunicación educativa (2ª ed.). Quito, Ecuador: CIESPAL.

Kaplún, Mario. (1999). Producción de programas de radio. El guion y la realización. Quito, Ecuador: Ediciones CIESPAL.

Kiewe, Amos. (1987). The College Radio Station: A model of experiential education. Trabajo presentado a 73rd Annual Meeting of the Speech Communication Association. Boston, Massachusetts, Estados Unidos. Recuperado de http://eric.ed.gov/?id=ED298547

Middleton, Chris. (2006). Creating digital music and sound. Burlington, Estados Unidos: Elsevier - Focal Press.

Montoya Ch., Alma D. y Villa Betancur, Lucelly. (2006). Radio Escolar: Una onda juvenil para una comunicación participativa. Bogotá, Colombia: Paulinas, Centro de Comunicación Social.

Pease Dreibelbis, María Angélica. (2011). Evaluación en el trabajo en equipo: aspectos a tomar en cuenta. En Blanco \& Negro, 2(1), 1-4. Recuperado de http://revistas.pucp.edu.pe/index.php/enblancoynegro/article/view/1483

Perona, Juan José. (2002). Radio escolar en Internet: un proyecto pedagógico para la era digital. Red digital: Revista de Tecnologías de la Información y Comunicación Educativas, (1). Recuperado de http://reddigital.cnice.mec.es/1/informes/infor perona res.html

Perona, Juan José. (2009). Edu-webs radiofónicas: experiencias españolas de educación en medios. Comunicar, 17(33), 107-114. Recuperado de http://www.redalyc.org/pdf/158/15812486013.pdf

Real Academia Española. (2014). Radiodifusión. Recuperado de http://dle.rae.es/srv/fetch?id=V1exuDP

Rodero, Emma. (2008). Educar a través de la radio. Signo y Pensamiento, 27(52), 97-109. Recuperado http://revistas.javeriana.edu.co/index.php/signoypensamiento/article/view/4581/3551

Sauls, Samuel. J. (abril, 2001). Understanding Your Media Outlet: An administrative Guide to the School Radio Station. Trabajo presentado en Annual Meeting of the Broadcast Education Association, Las Vegas, Estados Unidos. Recuperado de http://eric.ed.gov/?id=ED458644

Sauls, Samuel. J. (2000). The Culture of American College Radio. Ames, Estados Unidos: lowa State University Press. 
Sauls, Samuel. J. (january, 1998). The role of alternative programming in college radio. Trabajo presentado en Annual Meeting of the Southwest/Texas Popular Culture Association / American Culture Association. Recuperado de http://files.eric.ed.gov/fulltext/ED416529.pdf

Sauls, Samuel. J. (september, 1995a). College Radio: 10 Points of Contention from the Management Perspective. Trabajo presentado en Texas Association of Broadcast Educators Fall Student /Faculty Conference, San Antonio, Texas, Estados Unidos. Recuperado de http://files.eric.ed.gov/fulltext/ED410628.pdf

Sauls, Samuel. J. (april, 1995b). College Radio. Trabajo presentado en Annual Joint Meetings of the Popular Culture Association/ American Culture Association, Philadelphia, Estados Unidos. Recuperado de http://files.eric.ed.gov/fulltext/ED385885.pdf

Szyszko, Nancy, Neri, Carlos y Cataldi, Zulma. (2010). La radio en la escuela media como agente participativo. Quaderns digitals: Revista de Nuevas Tecnologías y Sociedad, (61), 1-15. Recuperado de http://dialnet.unirioja.es/servlet/articulo?codigo=3144263

Van der Graaf, Livia. (2001). Radios estudiantiles europeas: Análisis comparativo de emisoras estudiantiles en Francia, Gran Bretaña, Bélgica y España. Documento inédito, San José, Costa Rica: Universidad de Amsterdam, Universidad de Costa Rica, Radio U.

Zabalza, Miguel. (2009). Diseño y desarrollo curricular. Madrid, España: Narcea, S.A. de Ediciones. 